\title{
Literature survey on 'Performance Evaluation of TCP Reno, SACK, and FACK Over WIMAX Network
}

\author{
Kirti Sapra \\ M.Tech, Computer Science \& Engg., Shree Siddhivinayak Group Of Institutions, Yamuna Nagar, India
}

\begin{abstract}
This paper mainly aims in the improvement of the congestion control mechanism over mobile WiMAX .Here, TCP sender side mechanisms are used to handle higher load, random loss of packets and retransmission timeouts in high delay networks in such a way as to keep the maximum size of congestion window, while keeping the control over congestion and retransmissions at a minimal level. The TCP proposed mechanisms are assessed against TCP Reno, TCP SACK and TCP FACK to see how they fair against congestion and higher offered load. Ns2 simulator has been selected as the simulation tool because of the ease of use of the graphical interface provided, extensive support of TCP and the free license availability.
\end{abstract}

Keywords: WiMax, TCP Reno, TCP SACK, TCP FACK, NS2 Simulator

\section{INTRODUCTION}

WIMAX (Worldwide Interoperability for Microwave Access) is a wireless communications standard designed for providing 30 to 40 megabits-per-second data rates, with 2011 update providing up to $1 \mathrm{Gbit} / \mathrm{s}$ for fixed stations. The name "WIMAX" was created by the WIMAX Forum, which came into existence in June 2001 to promote conformity and interoperability of the standard. The forum describes WIMAX as "a standards-based technology enabling the delivery of last mile wireless broadband access as an alternative to DSL \& cable". WIMAX refers to interoperable attainments of the IEEE 802.16 family for wireless-networks standards ratified by WIMAX Forum. WIMAX Forum certification allows vendors to sell fixed or mobile products as WIMAX certified, hence, ensuring a level of interoperability with other certified products, to find whether they fit into the same profile.

The original IEEE 802.16 standard (now called "Fixed WIMAX") was advertised in 2001. WIMAX adopted some of its technology from WiBro, a service marketed in Korea. Mobile WIMAX (based on 802.16e-2005) is the revision that was deployed in many countries, and basis of forthcoming revisions such as ' $802.16 \mathrm{~m}-2011$ '.WIMAX is sometimes referred to as "Wi-Fi. on steroids" and can be used for lots of applications along with broadband connections, cellular back-haul, hotspots, etc.

It is like $\mathrm{Wi}-\mathrm{Fi}$, but it can enable convention at much greater distances. WIMAX is called the succeeding generation broadband wireless technology which tenders high speed, secure, sophisticate and last mile broadband services along with a cellular back haul and Wi-Fi hotspots.

The IEEE 802.16e air interface based on Orthogonal Frequency Division Multiple Access (OFDMA) whose main aim is to give better performance in non-line-of-sight ambience. IEEE 802.16e introduced scalable channel bandwidth up to $20 \mathrm{MHz}$, Multiple Input Multiple Output (MIMO) and AMC enabled 802.16e technology to support peak Downlink (DL) data rates up to $63 \mathrm{Mbps}$ in a 20 $\mathrm{MHz}$ channel through Scalable OFDMA (S-OFDMA) system .

\section{Fixed vs MobiLe WIMAX}

There are certain differences between Fixed WIMAX and Mobile WIMAX. 802.16d (Rev 2004) is known as Fixed WIMAX and 802.16e standard is referred as MobileWIMAX. The 802.16d standard provides support for fixed and drifting applications whereas 802.16e provides support for fixed, drifting, mobile and portable applications. The 802.16e carries all the features of $802.16 \mathrm{~d}$ standard besides new characterization that enables full mobility at fast speed, better QoS performance and power control but $802.16 \mathrm{e}$ devices are not congruent with 802.16d base stations as 802.16e are based on TDD whereas $802.16 \mathrm{~d}$ is based on FDD. Due to other compatibility issues with current networks, $802.16 \mathrm{e}$ has adopted S-OFDMA and 2048-FFT size. The purpose of mobile WIMAX is to support roaming capability and handover between Mobile Station (MS) and Base Station (BS).

\section{III.FEATURES OF WIMAX}

There are certain features of WIMAX those are making it popular day by day. Important features of WIMAX are described below:

\section{A. Interoperability}

The IEEE 802.16 standard is universally accepted and the standard is maintained and certified by WIMAX forum which covers stable, portable and mobile deployments and giving the user the freedom to choose their product from different certified vendors and use it in different stable, portable or mobile networks. 


\section{B. Mobility}

WIMAX offers immense mobility especially IEEE 802.16e-2005 as it adopted SOFDMA (Scalable Orthogonal Frequency Division Multiple Access) as a modulation technique and MIMO (Multiple Input Multiple Output) in its physical layer.

\section{Quality of Service}

Quality of Service (QoS) refers to the collective effect of service perceived by the end-users. Actually it refers to some particular requirements such as output, packet error rate, delay, and jitters etc. The wireless network must provide support to a variety of applications e.g.voice, data, video, and multimedia.

\section{Last Mile Connectivity}

Wireless network ingresses via DSL, T1-line or cable infrastructure are not available especially in rural areas. These connections have more restrictions which can be solved by WIMAX standards

\section{E. Accessibility}

To get high speed network connectivity, only necessary thing is to become a endorser of WIMAX service providers, then, they will provide a hardware that is very easy to install. Most of time hardware gets connected through USB ports or Ethernet and the connection may be made by clicking button.

\section{WIMAX ARCHITECTURE}

WIMAX architecture comprises of several components but the basic two components are BS and SS. Other constituents are MS, ASN, CSN and CSN-GW etc. WIMAX Forum's Network Working Group (NWG) has developed a network reference model according to the IEEE 802.16e-2005 air interface to assure the objectives of WIMAX are achieved. To support fixed, drifting and mobile WIMAX network, the network reference model can be reasonably divided into three parts .

\section{A. Mobile Station (MS)}

It is for the end user to access the mobile network and is a portable station able to move to wide areas and perform data and voice communication. It has all the required user equipments such as antenna, amplifier, transmitter, receiver and software needed to perform the wireless communication. GSM, FDMA devices etc are the examples of Mobile station.

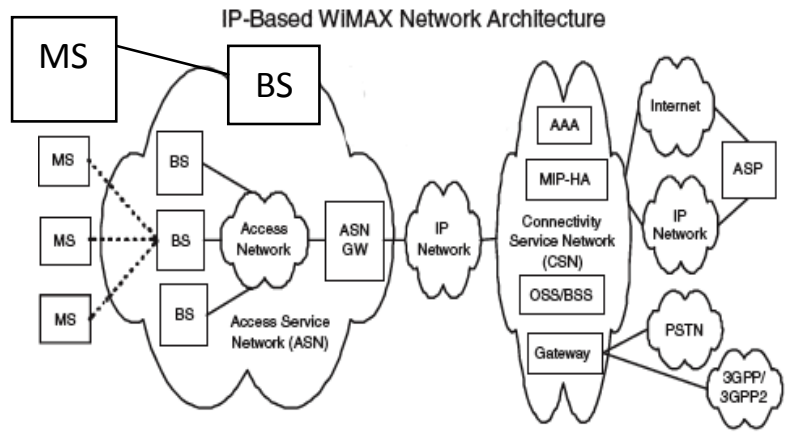

Fig .1 Architecture of Wimax

\section{B. Access Service Network (ASN)}

It is retained by NAP, formed with one or several base stations and ASN gateways (ASN-GW) which creates radio access network. It provides all the accessible services with full mobility and adequate scalability. Its ASN-GW controls the access in the network and coordinates between data and networking elements.

\section{Connectivity Service Network (CSN)}

Provides IP connectivity to the Internet or other public or collaborative networks. It also applies per user policy, address \& location management between ASNs \& ensures QoS, roving and security.

\section{SUPPORTEd BAND OF FREQUENCY}

The IEEE 802.16 supported licensed and unlicensed bands of interest are as follows:

\section{A . 10-66 GHz licensed band}

In this frequency band, due to lessened wave length, line of sight operation is required and as a result the effect of multipath propagation is evaded. The standard promises to provide data rates up to $120 \mathrm{Mb} / \mathrm{s}$ in this frequency band.The abundant availability of bandwidth is also another reason to operate in this frequency range.

\section{B. 2-11GHz licensed and licensed exempt}

In this frequency bands, both licensed and licensed absolved bands are consigned. Additional physical functionality supports have been introduced to operate in Near LOS and NLOS environment and to mitigate the effect of multipath proliferation. In fact, many of the IEEE 802.16 PHY's most beneficial capabilities are found in this frequency range. Operation in licensed absolve band experiences additional interference and coexistence issue. The PHY and MAC address mechanism like dynamic frequency selection (DFS) to detect and avoid interference (for licensed exempt band).

\section{Literature REVIEW}

Chen et al[1]. analyse the TCP performance in multi hop wireless networks based on the injected traffic and the control traffic. Infused traffic is mostly dependent on the optimal congestion window size which is proposed to be amid of $n / 3$ and $n / 2$, where $n$ is the total number of hops. Setting a fixed cwnd lessens the applicability of TCP. Therefore the literature focuses more on reducing the control traffic to increase TCP throughput.

Due to the elementary MAC layer, the transmission overhead time for longer data packets and ACK packets are almost of the same order of magnitude. The basic 802.11 data transfer in MAC Layer. Assuming a basic rate of $1 \mathrm{Mbps}$ (used for control traffic) and a data rate of 11 Mbps, the overhead transmission rate is 3 SIFS+RTS+CTS+ACKmac $=414 \mu \mathrm{s}$. Based on the above calculation time to send a 40 byte ACKtcp and 1040 byte data packets are $443 \mu$ s and $1170 \mu$ s respectively. To reduce ACKs, the strategy of using large ACK delay timeout does not work well always. If the congestion window is small, the receiver waits for ACK delay timeout to send the ACK, which leads to underutilization of the network. 
The literature proposes TCP sender to put the current and it has been found that an optimum value of QoS value of congestion window (cwnd) in the option field of parameters is obtained with increasing number of mobile TCP header, thereby notifying the receiver about its nodes for WiMax Network.

congestion window size. When the receiver calculates that the received but unacknowledged packets equal cwnd, it knows that the sender is awaiting for an ACK to proceed with sending the remaining packets and sends an ACK immediately. These self-triggered ACKs reduce the need to send ACK for every data segment and thereby reduce the control traffic, which in turn leads to elevated TCP throughput performance.

The authors[2] propose a receiver-aided mechanism in which the TCP receiver monitors the contention state of the connection and accordingly informs the TCP sender about it via ACK mechanism. TCP receiver uses end-toend delay as contention criteria.

Ruhani Ab Rahman et al[3]. presented an analysis on those routing protocols especially designed for wireless networks. A study and comparison on the pursuance of three routing protocols (AODV, DSR, and DSDV) for Mobile WiMAX climate is done. An presumption of each subscriber station has its routing capabilities within its own network is prepared. The performance factors includes Packet Delivery fraction (PDF), Throughput, End to End Delay, and number of packet dropped were diagnosed. The study used NS2 simulator for the comparison on the performance analysis. Successfully results observed that AODV protocol surpasses the DSR and DSDV.

In [4] Tarik Anouari et al. investigate the performances of the most common VoIP codecs, which are G.711, G.723.1 and G.729 over a WiMAX network using various service classes and NOAH as a transport protocol. Voice Over Internet Protocol is a promising new technology which provides access to voice communication over internet protocol based network, it becomes an substitute to public switched telephone networks due to its capability of transmission of voice as packets over IP networks. Therefore VoIP is largely outraged of delay and hence it needs a high priority transmission. protocol . To analyse the QoS parameters, network simulator ns-2 was used. Various parameters that actuate QoS of real life usage scenarios and traffic flows of applications is analysed. The aim is to compare different types of service classes with respect to the QoS parameters, e.g. throughput, average jitter and average delay.

Vikram Mehta et.al[5] focuses on analysing essential QoS parameters for Wimax Network. Essential QoS factors like delay, Jitter, Packet delivery Ratio (PLR), Packet Loss Ratio (PLR) and throughput have been calculated for 500 mobile nodes in a WiMax network. AODV protocol has been chosen as a routing protocol because of its ability to perform well under highly mobile and random conditions. MATLAB software version R2011 was used for creating WiMax network architecture and Regression analysis is done for each of the QoS parameter.The results help in critically analysing QoS parameters for WIMAX Network

\section{CONCLUSION}

WiMAX suffers from some issues like bandwidth constraints and limited power of mobile devices and some other are packet"s random loss of packets and retransmission interlude(with given load) in high delay networks that leads to congestion. So, TCP sender side mechanisms are used to handle these higher purposed load, random loss of packets and retransmission interludes in high delay networks in such a way as to keep congestion window at a maximum size, while keeping the congestion under control and keep retransmissions at a minimum level. Most of the schemes mentioned above clearly lacks in handling this and some other issues. Therefore there is definitely a need of congestion avoidance algorithms that can offer a better QoS improvement in WiMAX. From the above discussion TCP Reno, TCP SACK and TCP FACK delivers effective throughput and packet delivery ratio. Here the TCP proposed mechanisms are appraised against TCP Reno, TCP SACK and TCP FACK to see how they are legitimate against congestion and high offered load in the network.

\section{REFERENCES}

[1] B. Chen, I. Marsic, R. Miller, "Issues and Improvements in TCP Performance over Multihop Wireless Networks", IEEE Sarnoff Symposium, 2008.

[2] C. Chen, H. Wang, XinWang, M. Li, A.O. Lim, "A Novel Receiver-aided Scheme for Improv-ing TCP Performance in Multihop Wireless Networks", in Proc. of Int. Conference on Communications and Mobile Computing, pp. 272-277, 2009.

[3] Ruhani Ab Rahman ,"Performance Analysis of Routing Protocol in WiMAX Network", IEEE International Conference on System Engineering and Technology,2011.

[4] Tarik Anouari ,"Performance Analysis of VoIP Traffic in WiMAX using various Service Classes”, International Journal of Computer Applications ,Volume 52- No.20, August 2012.

[5] Vikram Mehta ,"Performance Analysis of QoS Parameters for Wimax Networks", International Journal of Engineering and Innovative Technology, Volume 1, Issue 5, May 2012 